\title{
Distribution of nuclei in equilibrium stellar matter from the free-energy density in a Wigner-Seitz cell
}

\author{
G. Grams, ${ }^{1}$ S. Giraud, ${ }^{2}$ A. F. Fantina, ${ }^{2}$ and F. Gulminelli ${ }^{3}$ \\ ${ }^{1}$ Universidade Federal de Santa Catarina, Brazil \\ ${ }^{2}$ Grand Accélérateur National d'Ions Lourds (GANIL), CEA/DRF - CNRS/IN2P3, Bvd Henri Becquerel, 14076 Caen, France \\ ${ }^{3}$ LPC (CNRS/ENSICAEN/Université de Caen Normandie), UMR6534, 14050 Caen Cédex, France
}

(Received 27 September 2017; revised manuscript received 22 December 2017; published 29 March 2018)

\begin{abstract}
The aim of the present study is to calculate the nuclear distribution associated at finite temperature to any given equation of state of stellar matter based on the Wigner-Seitz approximation, for direct applications in core-collapse simulations. The Gibbs free energy of the different configurations is explicitly calculated, with special care devoted to the calculation of rearrangement terms, ensuring thermodynamic consistency. The formalism is illustrated with two different applications. First, we work out the nuclear statistical equilibrium cluster distribution for the Lattimer and Swesty equation of state, widely employed in supernova simulations. Secondly, we explore the effect of including shell structure, and consider realistic nuclear mass tables from the Brussels-Montreal Hartree-Fock-Bogoliubov model (specifically, HFB-24). We show that the whole collapse trajectory is dominated by magic nuclei, with extremely spread and even bimodal distributions of the cluster probability around magic numbers, demonstrating the importance of cluster distributions with realistic mass models in core-collapse simulations. Simple analytical expressions are given, allowing further applications of the method to any relativistic or nonrelativistic subsaturation equation of state.
\end{abstract}

DOI: 10.1103/PhysRevC.97.035807

\section{INTRODUCTION}

Most of the methods used to calculate the equation of state (EoS) in the (proto)neutron-star crust and in supernova cores rely on the Wigner-Seitz (WS) approximation. In this framework, matter is divided into cells, each one charge neutral, with a nucleus at the center, surrounded by a gas of free nucleons (neutrons and, at finite temperature, free protons) and electrons. The standard way to calculate the EoS is then to minimize the (free) energy of the system with respect to the atomic and mass number of the nucleus, the volume of the cell (or its radius), and the free nucleon densities, under baryon number and charge conservation (see, e.g., the pioneer works of Refs. [1,2]). If additional structures, like the so-called "pasta" phases, are included, the minimization is also performed on the shape of the cell (see, e.g., the pioneer works of Refs. [3,4]).

Within this WS hypothesis, that is within the single nucleus approximation (SNA), different models to calculate the EoS of clustered matter at finite temperature have been developed, using the (compressible) liquid-drop method (see, e.g., Refs. [5-9]), the (extended) Thomas-Fermi approach (see, e.g., Refs. [10-21]), or more microscopic self-consistent mean-field Hartree-Fock and Hartree-Fock-Bogoliubov models (see, e.g., Refs. [22-27]); see also Ref. [28] for a review. In particular, the two currently most employed EoSs in supernova simulations, namely the Lattimer and Swesty (LS) [8] and the Shen et al. $[12,14]$ EoSs, both make use of this WS concept of a unique configuration associated to each thermodynamic condition, given by the baryon density $n_{B}$, the temperature $T$, and the proton fraction $Y_{p}$ (or, equivalently, the electron fraction $Y_{e}$; because of charge conservation, $Y_{e}=Y_{p}$ ). While for the physics of "cold" (catalyzed) neutron stars this assumption is correct (in the absence of phase transitions), at finite temperature the approximation of a unique configuration, which is the most favorable from the thermodynamic point of view, has to be seen as an average way to account for the properties of a statistical distribution. Despite this approximation does not change considerably the thermodynamic properties of matter [29], quantitative differences arise in the matter composition, in particular concerning the contribution of light and intermediate mass nuclei. In turn, this impacts the calculation of reaction rates on individual nuclei thus affecting the dynamical processes depending on these rates (see, e.g., Refs. [30-33]).

Recently, efforts have been made to construct EoSs for supernova matter including an ensemble of nuclei, based on the (extended) nuclear statistical equilibrium (NSE) approach, starting with the pioneer works of El Eid, Hillebrandt et al. [34,35] (see, e.g., Refs. [36-49]). While standard NSE-based models treat the matter constituents as a mixture of noninteracting ideal gases governed by the Saha equation, neglecting interactions and in-medium effects (see, e.g., the classical textbooks [50,51]), in extended NSE approaches the distribution of clusters is obtained self-consistently under conditions of statistical equilibrium and interactions are taken into account. Indeed, in-medium corrections are known to be particularly important at high density. The way of treating these corrections, as well as other model assumptions such as the choice of the nuclear masses and/or level densities, are also responsible for affecting the thermodynamic quantities and the nuclear composition predicted by different NSE models (see, e.g., Ref. [52]). Moreover, a limitation of the current available EoSs based on the NSE model is that they are given in the form of 
tables, thus systematic investigations in numerical simulations varying one or the other nuclear input are difficult to perform.

In this work, we derive a general formalism, based on the work of Ref. [47], to include an extended NSE model at subsaturation density and finite temperature in any approach, either nonrelativistic or relativistic, based on the WS cell approximation, starting from an arbitrary free energy density and including explicit cluster degrees of freedom. In Sec. II we introduce the formalism and the particular application to the LS EoS is presented in Sec. III. Details on the formal derivations are given in Appendices A and B. In Sec. IV we discuss the results obtained employing this formalism in a perturbative way, on the top of the LS EoS, using both the original compressible liquid-drop model and including more microscopic nuclear mass table for the calculation of the nuclear binding energies (Sec. IV A). Finally, in Sec. V, we draw our conclusions and future outlooks.

\section{NSE FROM A WIGNER-SEITZ FREE ENERGY}

Let us consider an arbitrary model giving the free energy in a WS cell, $F_{\mathrm{WS}}$, as a function of the variational parameters. While at zero temperature a unique realization of the WS cell is expected, at finite temperature different realizations of the WS cell are possible (see, e.g., Ref. [47]). If we consider a very large volume $V \rightarrow \infty$, containing a large number of WS cells, each one with volume $V_{C}^{(j)}$, such that

$$
V(k)=\sum_{j} N_{j}(k) V_{C}^{(j)},
$$

$N_{j}(k)$ being the number of occurrences of the cell $(j)$ in a configuration $k=\left\{N_{j}(k), j=1, \ldots, \infty\right\}$, the total free energy of the system is given by

$$
F_{\mathrm{tot}}(k)=\sum_{j} N_{j}(k) F_{\mathrm{WS}}^{(j)} .
$$

Here, $F_{\mathrm{WS}}=F_{\mathrm{WS}}\left(\left\{\mathbf{x}_{k}\right\}\right)$ is the cell free energy, depending on a set $\left\{\mathbf{x}_{k}\right\}$ of variational variables. In the case of an EoS model with cluster degrees of freedom, a typical choice for this set is $\left\{A, Z, n_{n g}, n_{p g}, V_{C}\right\}, A(Z)$ being the number of baryons (protons) in the cluster and $n_{n g}\left(n_{p g}\right)$ the number density of neutrons (protons) in the gas, so that we have $F_{\mathrm{WS}}=F_{\mathrm{WS}}\left(A, Z, n_{n g}, n_{p g}, V_{C}\right)$. The notation $F_{\mathrm{WS}}^{(j)}$ thus indicates the value of $F_{\mathrm{WS}}$ when the variational variables take the values characteristic of the cell $(j), F_{\mathrm{WS}}^{(j)}=$ $F_{\mathrm{WS}}\left(A^{(j)}, Z^{(j)}, n_{n g}^{(j)}, n_{p g}^{(j)}, V_{C}^{(j)}\right)$. Other choices for the $\left\{\mathbf{x}_{k}\right\}$ set are also possible, and we will explicitly express the problem in terms of the variational variables of the LS EoS subsequently in the paper. In the case of EoSs based on the density functional theory, where the variational variables are the single-particle wave functions, the definition of these cluster variables has to be derived from the model. Typically, one can take $n_{q g}=$ $n_{q g}\left(r=R_{C}\right)(q=n, p), A=V_{C}\left(n_{B}-n_{n g}-n_{p g}\right)$, and $Z=$ $V_{C}\left(n_{B} Y_{p}-n_{p g}\right)$, with $R_{C}$ the cell radius.

The WS cell free energy, $F_{\mathrm{WS}}$, reads

$$
F_{\mathrm{WS}}=F_{\text {nuc }}+F_{g}+F_{\text {lept }}+F_{\gamma}
$$

and accounts for the contribution of the cluster, the gas, the leptons (electron and possibly positrons and neutrinos), and the photons, respectively. Leptons and photons can be described as a uniform (relativistic) gas, and can be treated separately (see, e.g., Refs. [8,53,54] for complete expressions). Therefore, from now on, we will focus only on the (cluster plus gas) baryon contribution, whose total free energy can be written as

$$
F_{\text {bar }}(k)=\sum_{j} N_{j}(k)\left[F_{\text {nuc, vac }}^{(j)}+\mathcal{F}_{g}^{(j)} V_{C}^{(j)}+\delta F^{(j)}\right],
$$

where $\mathcal{F}_{g}$ is the free energy density of homogeneous nuclear matter with neutron and proton density $\left(n_{n g}, n_{p g}\right), F_{\text {nuc, vac }}$ is the free energy of a cluster $(A, Z)$ in vacuum, and $\delta F$ accounts for the in-medium contributions. The latter may include Coulomb screening and surface terms, and possibly contributions due to the excluded-volume approximation if the original model employs this scheme. Including all in-medium effects in the cluster contribution, one can define $F_{\text {nuc }}=F_{\text {nuc,vac }}+\delta F$. In the SNA, for each thermodynamic condition $\left(n_{B}, T, Y_{p}\right)$, the variational variables are determined by minimizing the total free energy density under the constraints of mass and charge conservation. The associated values of $A$ and $Z$ have therefore to be interpreted as the nucleus contained in the most probable cell among the different possibilities $(j)$ at the given thermodynamic conditions, and will be noted $\left(\langle A\rangle_{j}=A_{\mathrm{SNA}},\langle Z\rangle_{j}=\right.$ $\left.Z_{\mathrm{SNA}}\right)$. However, in a full thermodynamic treatment of Eq. (4), the variational variables are given by the set $N_{j}(k)$, and the variation gives the average occupation $\left\langle N_{j}\right\rangle_{k}$ over the different configurations $k$, or equivalently the probability $\left\langle p_{j}\right\rangle_{k} \equiv p_{j}$. As a result, one does not get a single $A=A_{\mathrm{SNA}}, Z=Z_{\mathrm{SNA}}$ value, but a distribution of possible $\left(A^{(j)}, Z^{(j)}\right)$ configurations with probability $p_{j}$.

In order to calculate the probability $p_{j}$, it is convenient to work in the grand-canonical ensemble. In this framework, one can construct the grand-canonical partition sum associated to the free energy $F_{\text {bar }}$, starting from the Gibbs free energy of the baryon component

$$
G_{\mathrm{bar}}(k)=F_{\mathrm{bar}}(k)-\sum_{j} N_{j}(k)\left(\mu_{n} N_{\mathrm{WS}}^{(j)}+\mu_{p} Z_{\mathrm{WS}}^{(j)}\right),
$$

where $\mu_{n}\left(\mu_{p}\right)$ is the neutron (proton) chemical potential. We refer to Appendix A for the proof that the neutron and proton chemical potentials correspond in fact to those of the gas, i.e., $\mu_{n}=\mu_{n g}$ and $\mu_{p}=\mu_{p g}$, and do not depend on the realization $(k)$. The total numbers of neutrons (protons) in the WS cell $(j), N_{\mathrm{WS}}^{(j)}\left(Z_{\mathrm{WS}}^{(j)}\right)$, are given by

$$
\begin{aligned}
& N_{\mathrm{WS}}^{(j)}=N^{(j)}+n_{n g} V_{C}^{(j)}, \\
& Z_{\mathrm{WS}}^{(j)}=Z^{(j)}+n_{p g} V_{C}^{(j)} .
\end{aligned}
$$

Notice that the gas densities do not depend on the cell $(j)$, since the gas is uniform over the whole volume. If this condition were not satisfied, discontinuities in the gas densities would arise at the cell interface. Therefore, also the chemical potentials of the gas, $\mu_{n}$ and $\mu_{p}$, do not depend on the cell $(j)$. The conservation equations, Eqs. (6),(7), have to be modified if the considered EoS employs excluded-volume effects. This is easily done by introducing the number of nucleons in the dense region of 
space, $N_{r}$ and $Z_{r}$, and by replacing in all equations $N \rightarrow N_{r}$ $n_{n g} V_{N}, Z \rightarrow Z_{r}-n_{p g} V_{N}$ (thus $A_{r}=N_{r}+Z_{r}$ ), with $V_{N}$ the cluster volume. This will be explicitly worked out in the next section on the example of the LS model.

Replacing the conservation equations into Eq. (5), we get

$$
\begin{aligned}
G_{\text {bar }}(k)= & \sum_{j} N_{j}(k)\left[F_{\text {nuc }}^{(j)}-\mu_{n} N^{(j)}-\mu_{p} Z^{(j)}\right] \\
& +V\left[\mathcal{F}_{g}-\mu_{n} n_{n g}-\mu_{p} n_{p g}\right] .
\end{aligned}
$$

In principle the gas densities $n_{n g}, n_{p g}$, though uniform throughout the total volume $V$, could depend on the realization $(k)$ through the occupation $N_{j}(k)$. It is however easy to check that this cannot be the case if we keep the hypothesis that each cell $(j)$ is electrically neutral, as it has to be to avoid Coulomb interactions between the different cells.

Equation (8) defines the Gibbs free energy of the cluster and the Gibbs free energy density of the gas

$$
\begin{aligned}
G_{\text {nuc }}^{(j)} & =F_{\text {nuc }}^{(j)}-\mu_{n} N^{(j)}-\mu_{p} Z^{(j)}, \\
\mathcal{G}_{g} & =\mathcal{F}_{g}-\mu_{n} n_{n g}-\mu_{p} n_{p g} .
\end{aligned}
$$

The explicit expressions of $\mathcal{F}_{g}$ and $F_{\text {nuc }}^{(j)}$ depend on the particular model employed. In particular, if the EoS is obtained through a density functional model, $F_{\text {nuc }}^{(j)}$ depends on the density. In that case, because of the baryon number and charge conservation laws,

$$
\begin{aligned}
& n_{B}=\frac{\sum_{j} N_{j}(k) A^{(j)}}{V}+n_{g}, \\
& n_{p}=\frac{\sum_{j} N_{j}(k) Z^{(j)}}{V}+n_{p g},
\end{aligned}
$$

$n_{p}$ being the total proton number density and $n_{g}=n_{n g}+$ $n_{p g}, F_{\text {nuc }}^{(j)}$ implicitly depends on $N_{j}(k)$ through its density dependence. This introduces a self-consistency problem, which does not arise for instance if $F_{\text {nuc }}^{(j)}$ is obtained through a standard (i.e., noncompressible) liquid-drop model without any density dependence. In the general case of a density-dependent free energy, a rearrangement term has to be added. The effective one-body equivalent free energy is given by

$$
\tilde{F}_{\text {nuc }}^{(j)}=\frac{\partial\left\langle F_{\text {bar }}\right\rangle_{k}}{\partial\left\langle N_{j}\right\rangle_{k}}=F_{\text {nuc }}^{(j)}+\left.\left\langle N_{j}\right\rangle_{k} \frac{\partial F_{\text {nuc }}^{(j)}}{\partial\left\langle N_{j}\right\rangle_{k}}\right|_{n_{n g}, n_{p g},\left\langle N_{i}\right\rangle_{k, i \neq j}}
$$

with $\left\langle F_{\mathrm{bar}}\right\rangle_{k}=F_{\mathrm{bar}}\left(\left\{\left\langle N_{j}\right\rangle_{k}\right\}\right)$. Notice that in the partial derivative the gas component does not appear, because the term $\sum_{j} N_{j}(k) \mathcal{F}_{g} V_{C}^{(j)}=V \mathcal{F}_{g}$ in Eq. (4) does not depend on $N_{j}(k)$, since the gas is homogeneous over the different cells.

The total effective Gibbs free energy of the baryon component becomes

$$
\tilde{G}_{\text {bar }}(k)=\sum_{j} N_{j}(k) \tilde{G}_{\text {nuc }}^{(j)}+\mathcal{G}_{g} V
$$

with

$$
\tilde{G}_{\text {nuc }}^{(j)}=\frac{\partial\left\langle G_{\mathrm{bar}}\right\rangle_{k}}{\partial\left\langle N_{j}\right\rangle_{k}}=G_{\text {nuc }}^{(j)}+\left.\left\langle N_{j}\right\rangle_{k} \frac{\partial G_{\text {nuc }}^{(j)}}{\partial\left\langle N_{j}\right\rangle_{k}}\right|_{n_{n g}, n_{p g},\left\langle N_{i}\right\rangle_{k, i \neq j}}
$$

Introducing the grand-canonical partition function associated to the independent cluster problem (plus gas),

$$
\tilde{\mathcal{Z}}_{\beta \mu_{n} \mu_{p}}=\sum_{k} \exp \left(-\beta \tilde{G}_{\mathrm{bar}}(k)\right),
$$

where $\beta=\left(k_{B} T\right)^{-1}, k_{B}$ being the Boltzmann constant, shows that in the thermodynamic limit the partition sum of the cluster, $\tilde{\mathcal{Z}}_{\text {nuc }}$, and that of the gas, $z_{\beta \mu_{n} \mu_{p}}$, can be factorized as

$$
\tilde{\mathcal{Z}}_{\beta \mu_{n} \mu_{p}}=\tilde{\mathcal{Z}}_{\text {nuc }}\left(z_{\beta \mu_{n} \mu_{p}}\right)^{V} \text {. }
$$

The partition sum of the cluster thus reads

$$
\tilde{\mathcal{Z}}_{\text {nuc }}=\sum_{k} \exp \left(-\beta \sum_{j} N_{j}(k) \tilde{G}_{\text {nuc }}^{(j)}\right),
$$

and, thanks to this factorization, one can follow the standard derivation of thermodynamics of an ideal classical gas for the cluster component:

$$
\begin{aligned}
\tilde{\mathcal{Z}}_{\text {nuc }} & =\prod_{j} \sum_{n=0}^{\infty} \frac{\left(\exp \left(-\beta \tilde{G}_{\text {nuc }}^{(j)}\right)\right)^{n}}{n !} \\
& =\prod_{j} \exp \left(w_{\beta \mu_{n} \mu_{p}}^{(j)}\right),
\end{aligned}
$$

where $w_{\beta \mu_{n} \mu_{p}}^{(j)} \equiv \exp \left(-\beta \tilde{G}_{\text {nuc }}^{(j)}\right)$. In this way, similarly to Eq. (110) in Ref. [47], one recovers a NSE-like expression for the cluster multiplicities

$$
\left\langle N_{j}\right\rangle_{k}=\frac{\partial\left(\ln \tilde{\mathcal{Z}}_{\text {nuc }}\right)}{\partial\left(\beta \mu_{j}\right)}=w_{\beta \mu_{n} \mu_{p}}^{(j)}=\exp \left(-\beta \tilde{G}_{\text {nuc }}^{(j)}\right),
$$

where $\mu_{j}=\left(\mu_{n}+\mu_{p}\right) A^{(j)}$. Finally, we can express the cluster probability as

$$
p_{j}=\frac{\left\langle N_{j}\right\rangle_{k}}{\sum_{j}\left\langle N_{j}\right\rangle_{k}}=\frac{\exp \left(-\beta \tilde{G}_{\text {nuc }}^{(j)}\right)}{\sum_{j} \exp \left(-\beta \tilde{G}_{\text {nuc }}^{(j)}\right)} .
$$

Note that the functional form of Eq. (20) is formally equivalent to the classical grand-canonical formulation (see, e.g., Refs. [51,55]). However, it has to be stressed that the correspondence between the canonical and grand-canonical formulation is only possible if one writes the Gibbs free energy as Eq. (15).

We turn now to the calculation of the rearrangement term. As already noticed in Sec. III C of Ref. [47], this term is expected to be small and was neglected in Ref. [47]. However, as we will show in Sec. III, the effect of the rearrangement term can be non-negligible and, indeed, this term is necessary to recover the correct SNA results in all density domains.

As we have already noticed, the dependence of $G_{\text {nuc }}^{(j)}$ on $\left\langle N_{j}\right\rangle_{k}$ arises from the dependence on the total density, which induces a constraint among the $\left\langle N_{j}\right\rangle_{k}$ variables, see Eq. (12). Because of the condition of charge neutrality in each cell, $n_{p}=$ $Z^{(j)} / V_{C}^{(j)}+n_{p g}$, this can be seen as a dependence on the cell volume $V_{C}^{(j)}$.

The rearrangement term can thus be expressed as

$$
\left\langle N_{j}\right\rangle_{k} \frac{\partial G_{\mathrm{nuc}}^{(j)}}{\partial\left\langle N_{j}\right\rangle_{k}}=-V_{C}^{(j)} \frac{\partial G_{\mathrm{nuc}}^{(j)}}{\partial V_{C}^{(j)}} \frac{\left\langle N_{j}\right\rangle_{k} V_{C}^{(j)}}{V} .
$$


We can see that $\tilde{G}_{\text {nuc }}^{(j)}$ explicitly depends on $\left\langle N_{j}\right\rangle_{k}$, meaning that Eq. (21) is in reality a self-consistent equation. Since the rearrangement correction is supposed to be small (we will quantify this statement in the next section on the specific example of the LS EoS), we make an approximation in order to avoid the complication of a self-consistent resolution of Eq. (21). We first remark from Eqs. (9) and (15) that $\tilde{G}_{\text {nuc }}^{(j)}$ should be proportional to $A^{(j)}$. We therefore single out the $A^{(j)}$ dependence from Eq. (22) and average the rest of the expression over the different cells. Using $V_{C}^{(j)}=A^{(j)} /\left(n_{B}-n_{g}\right)$, and observing that $\left\langle\left\langle N_{j}\right\rangle_{k} V_{C}^{(j)}\right\rangle_{j}=V$, we get

$$
\left\langle N_{j}\right\rangle_{k} \frac{\partial G_{\mathrm{nuc}}^{(j)}}{\partial\left\langle N_{j}\right\rangle_{k}} \approx-V_{C}^{(j)}\left\langle\frac{\partial G_{\mathrm{nuc}}^{(j)}}{\partial V_{C}^{(j)}}\right\rangle_{j}=-\frac{A^{(j)}}{n_{B}-n_{g}}\left\langle\frac{\partial G_{\mathrm{nuc}}^{(j)}}{\partial V_{C}^{(j)}}\right\rangle_{j} .
$$

In this expression, $\left\langle\partial G_{\text {nuc }}^{(j)} / \partial V_{C}^{(j)}\right\rangle_{j}$ is the most probable value of the function $\partial G_{\text {nuc }} / \partial V_{C}$ among the different cells, and should be taken from the original SNA model.

The final expression for the effective Gibbs energy, Eq. (15), is then

$$
\tilde{G}_{\mathrm{nuc}}^{(j)}=G_{\mathrm{nuc}}^{(j)}-\frac{A^{(j)}}{n_{B}-n_{g}}\left(\frac{\partial G_{\mathrm{nuc}}}{\partial V_{C}}\right)_{\mathrm{SNA}},
$$

which together with Eqs. (9) and (21) completes the model.

With the choice $\left\{\mathbf{x}_{k}\right\}=\left\{A, Z, n_{n g}, n_{p g}, V_{C}\right\}$, Eq. (21) becomes a probability distribution for the different clusters of size $(A, Z)$ as

$$
p(A, Z)=\frac{\exp \left(-\beta \tilde{G}_{\mathrm{nuc}}(A, Z)\right)}{\sum_{A, Z} \exp \left(-\beta \tilde{G}_{\mathrm{nuc}}(A, Z)\right)},
$$

where the gas variables $n_{n g}, n_{p g}$ are taken from the SNA model, and the cell volume is obtained for each cluster from the neutrality condition

$$
V_{C}=\frac{Z}{n_{B} Y_{p}-n_{p g}} .
$$

It has been already discussed in Ref. [47] that one can recover the SNA expression by minimizing the free energy density under the mass and charge conservation constraints, by using an auxiliary function. We will not redo the general demonstration here, but we will apply this procedure in Appendix B for the specific case of the LS EoS, thus showing that the most probable cluster which maximizes $p_{j}$ (or, equivalently, minimizes $\tilde{G}_{\text {nuc }}$ ), coincides with the (unique) cluster predicted by the LS equations.

\section{DISTRIBUTION OF NUCLEI FROM THE LATTIMER AND SWESTY EQUATION OF STATE}

The LS EoS [8] models matter as a mixture of a single species of heavy nuclei (representing the average of a full ensemble of heavy nuclei), $\alpha$ particles (representative of light nuclei), and free neutrons and protons, immersed in a uniform noninteracting gas of leptons and photons. At low density, nuclei are considered as spherical, at the center of a charge-neutral spherical cell and surrounded by a less dense vapor of neutrons, protons, and $\alpha$ particles, as well as electrons, positrons, and photons, in the WS approximation. For nucleons, either inside the nucleus or in the gas, a simplified nucleon-nucleon interaction of Skyrme type is employed. $\alpha$ particles are described as hard spheres of volume $v_{\alpha}=24 \mathrm{fm}^{3}$ obeying an ideal Boltzmann gas statistics. Interaction between heavy nuclei and the gas of $\alpha$ particles and nucleons are treated in an excluded-volume approach. With increasing density, shape deformations of nuclei (nonspherical nuclei and bubble phases) are taken into account by modifying the Coulomb and surface energies, and the transition to uniform matter is described by a Maxwell construction (see Ref. [8] for more details). The original LS EoS routine, which is also used in the present paper, is available at http://www.astro.sunysb.edu/dswesty/lseos.html (more recent EoSs in tabular form are given at http://www.astro.sunysb.edu/lattimer/EOS/main.html). There exist three parametrizations of the LS EoS routine, according to the value of the incompressibility of the underlying nuclear interaction $(K=180,220$, and $375 \mathrm{MeV})$. For the numerical applications done in the present paper, we use the parametrization with $K=220 \mathrm{MeV}$. Note that a minor correction has been done with respect to the original code. Indeed, it has been recognized (see, e.g., Ref. [56]) that the original LS EoS underestimated the fraction of $\alpha$ particles, because the $\alpha$ particle binding energy, $B_{\alpha}$, has to be measured with respect to the neutron mass, as all other energies (see, e.g., Ref. [57]).

For every baryon density $n_{B}$, temperature $T$, and electron (or proton) fraction $Y_{e}$, the EoS is obtained by minimizing the cell Helmoltz free energy density. As discussed in Sec. II, the nonbaryonic components of the free energy density can be treated separately, and have no role in the equilibrium equations. The chosen seven independent variables of the system in the LS EoS are $\left\{x_{k}\right\}=\left\{n_{i}, x_{i}, u, r_{N}, n_{\alpha}, n_{n o}, n_{p o}\right\}$, where $n_{i}$ and $x_{i}$ are the nucleon density and proton fraction inside the nucleus, respectively, $u=V_{N} / V_{C}$ is the fraction of the WS cell volume occupied by nuclei $\left(V_{N}\right.$ being the volume of the nucleus), $r_{N}$ is the nuclear radius (such that $\left.V_{N}=4 \pi r_{N}^{3} / 3\right), n_{\alpha}, n_{n o}$, and $n_{p o}$ are the number density of the $\alpha$ particles, and of the neutrons and protons in the outside gas, respectively. However, because of charge neutrality and baryon conservation, the number of independent variables is reduced to five. The minimization is done by taking partial derivatives of the free energy with respect to the remaining five independent variables, thus yielding a system of equilibrium equations (see Eqs. (3.2) in Ref. [8]). This procedure is equivalent to the minimization of the free energy density with respect to all variational variables applying the canonical constraints as Lagrange multipliers, as shown in Appendix B.

However, to construct a NSE with a distribution of nuclei starting from the free energy density of the LS EoS, one has to work in the grand-canonical ensemble. Following the procedure explained in Sec. II and in Appendix A, and noting that the gas contribution to the free energy density can be divided into the contribution coming from $\alpha$ particles, $\mathcal{F}_{\alpha}$, and that of the nucleons outside the nucleus, $\mathcal{F}_{o}$, the Gibbs free energy of the cluster, Eq. (9), and the Gibbs free energy density of the gas (that of the nucleons in the gas, $\mathcal{G}_{o}$, and that 
of $\alpha$ particles, $\left.\mathcal{G}_{\alpha}\right)$, Eq. (10), in each cell $(j)$, read

$$
\begin{aligned}
G_{\text {nuc }}^{(j)} & =F_{\text {nuc }}^{(j)}-\mu_{n} N^{(j)}-\mu_{p} Z^{(j)}, \\
\mathcal{G}_{o} & =\mathcal{F}_{o}-\mu_{n} n_{n o}-\mu_{p} n_{p o}, \\
\mathcal{G}_{\alpha} & =\mathcal{F}_{\alpha}-\mu_{\alpha} n_{\alpha},
\end{aligned}
$$

where $\mu_{\alpha}$ is the chemical potential of $\alpha$ particles. Using the LS variables, ${ }^{1}$ the free energy of the cluster, $F_{\text {nuc }}$, that contains the in-medium corrections (including the excluded-volume terms), can be written as

$$
\begin{aligned}
F_{\text {nuc }}= & V_{N}\left[n_{i}\left(f_{b}+f_{s c}+f_{H}\right)-f_{\alpha} n_{\alpha}-\left(1-n_{\alpha} v_{\alpha}\right) n_{o} f_{o}\right] \\
& -n_{\alpha} v_{\alpha} n_{o} f_{o} V_{C},
\end{aligned}
$$

where $f_{b}, f_{s c}$, and $f_{H}$ are the bulk, the finite size (surface and Coulomb), and the translational contributions to the free energy per baryon, respectively, and $n_{o}=n_{n o}+n_{p o}$. In this expression, the dependence on the cell is given by the cluster variables, i.e., $\left\{n_{i}, x_{i}, V_{N}, u\right\}=\left\{n_{i}^{(j)}, x_{i}^{(j)}, V_{N}^{(j)}, u^{(j)}\right\}$ and the cell volume $V_{C}=V_{C}^{(j)}$, since $\alpha$ particles and free nucleons are assumed uniform in the different cells.

The cluster neutron and proton numbers in each cell $(j), N^{(j)}$ and $Z^{(j)}$, fulfilling the conservation equations

$$
\begin{aligned}
& n_{B}=\frac{A}{V_{C}}+4 n_{\alpha}+n_{o}, \\
& n_{p}=\frac{Z}{V_{C}}+2 n_{\alpha}+n_{p o},
\end{aligned}
$$

are given by

$$
\begin{aligned}
& N=V_{N}\left[\left(1-x_{i}\right) n_{i}-n_{n o}-2 n_{\alpha}-n_{n o} v_{\alpha} n_{\alpha} \frac{1-u}{u}\right], \\
& Z=V_{N}\left[x_{i} n_{i}-n_{p o}-2 n_{\alpha}-n_{p o} v_{\alpha} n_{\alpha} \frac{1-u}{u}\right]
\end{aligned}
$$

with $A=N+Z$. Notice that because of the excluded volume, these numbers do not represent the number of nucleons in the dense phase, the latters being given, for each cell $(j)$, by $A_{r}=$ $n_{i} V_{N}, Z_{r}=x_{i} n_{i} V_{N}$.

To calculate the rearrangement term, we work out explicitly the expression of Eq. (22). The charge conservation law is written for LS variables as

$$
n_{p}=\frac{\sum_{j}\left\langle N_{j}\right\rangle_{k} V_{N}^{(j)}\left(x_{i}^{(j)} n_{i}^{(j)}-n_{p g}\right)}{V}+n_{p g}
$$

with the short-hand notation $n_{p g}=2 n_{\alpha}+n_{p o}\left(1-n_{\alpha} v_{\alpha}\right)$.

As in the general derivation, the definition of $n_{p}$, Eq. (35), breaks the independence among the cell occupations $N_{j}(k)$. In turn, this induces a self-consistency problem because the Gibbs free energy $G_{\text {nuc }}$ depends on $n_{p}$ through the Coulomb and surface term. Writing the charge conservation in the single WS cell:

$$
n_{p}=u\left(x_{i} n_{i}-n_{p g}\right)+n_{p g}
$$

\footnotetext{
${ }^{1}$ Note that, unlike Ref. [8], we use capital letters for the energy, e.g., $F$ for the free energy, $\mathcal{F}$ for the free energy density, and small letters for the energy per baryon, e.g., $f$ for the free energy per baryon.
}

we can see that it is more convenient to change variables in Eq. (22) from $V_{C}$ to $u=V_{N} / V_{C}$.

The rearrangement term then reads

$$
\left\langle N_{j}\right\rangle_{k} \frac{\partial G_{\mathrm{nuc}}^{(j)}}{\partial\left\langle N_{j}\right\rangle_{k}}=\left\langle N_{j}\right\rangle_{k} \frac{\partial G_{\mathrm{nuc}}^{(j)}}{\partial u^{(j)}} \frac{\partial u^{(j)}}{\partial n_{p}} \frac{\partial n_{p}}{\partial\left\langle N_{j}\right\rangle_{k}} .
$$

Using $n_{i}=A_{r} / V_{N}$, we can single the dependence on the cluster size out of this expression, and average the rest of this term with respect to the different cells. The one-body Gibbs free energy, Eq. (24), can finally be written as

$$
\begin{aligned}
\tilde{G}_{\mathrm{nuc}}(A, Z) & =G_{\mathrm{nuc}}(A, Z)+A_{r}^{(j)}\left\langle\frac{\left\langle N_{j}\right\rangle_{k}}{n_{i}^{(j)} V} \frac{\partial G_{\mathrm{nuc}}}{\partial u}\right\rangle_{j} \\
& =G_{\mathrm{nuc}}(A, Z)+A_{r}^{(j)}\left(\frac{u}{n_{i} V_{N}} \frac{\partial G_{\mathrm{nuc}}}{\partial u}\right)_{\mathrm{SNA}},
\end{aligned}
$$

where the quantities within the brackets \langle\rangle$_{j}$ have to be calculated for the most probable nucleus as given by the standard solution of the LS equations (see Appendix B).

To verify the thermodynamic consistency of the approach we can check that the most probable configuration given by the NSE coincides with the LS representative cluster. To this aim, one has to minimize the exponent in Eq. (20), i.e., the Gibbs free energy, Eq. (38). For a better readability of the paper, the LS equations defining the representative configuration in the SNA are explicitly rederived in Appendix B.

Note that, in the grand-canonical ensemble, the density of the outside gas, i.e., that of the free neutrons and protons and that of alpha particles, are fixed by their chemicals potentials (see Appendix A):

$$
n_{q o}=\frac{\partial g_{o}}{\partial \mu_{q o}} ; \quad n_{\alpha}=\frac{\partial g_{\alpha}}{\partial \mu_{\alpha}},
$$

where $q=n, p$ and $g_{o}\left(g_{\alpha}\right)$ is the Gibbs free energy per baryon of the nucleons in the outside gas (of the $\alpha$ particles). Moreover, we further notice that the model requires $n_{B}$ [as well as $n_{p}$, see Eq. (32)] to be the same in each WS cell. Therefore, different ions in the cell, in the NSE approach, can have different baryon number $A$ (the gas baryon number being uniform in the whole volume and the same in each cell) and different volume $V_{N}$, keeping $n_{B}$, Eq. (31), constant. Equation (31) thus fixes $V_{C}$, or equivalently $u$. With these constraints, Eqs. (31), (32), and (39), the seven independent variables on which the minimization has to be done are reduced to three, namely, $\left(n_{i}, x_{i}, r_{N}\right)$. Calculating the partial derivatives of the effective Gibbs free energy yields

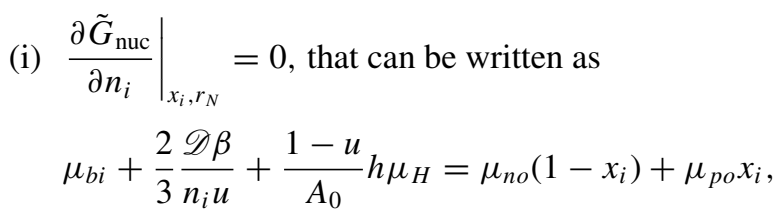

where $\mu_{b i}=\left(1-x_{i}\right) \mu_{n i}+x_{i} \mu_{p i}, \mu_{n i}$ and $\mu_{p i}$ are the chemical potentials of the neutrons and protons inside the nucleus, and $\beta \mathscr{D}=\mathcal{F}_{c}+\mathcal{F}_{s}$ is the sum of the Coulomb and surface free energy density (see Eq. (2.39) in [8]). The constant $A_{0}$ in the translational free energy term has been taken equal to 60 in [8], $h \equiv$ 

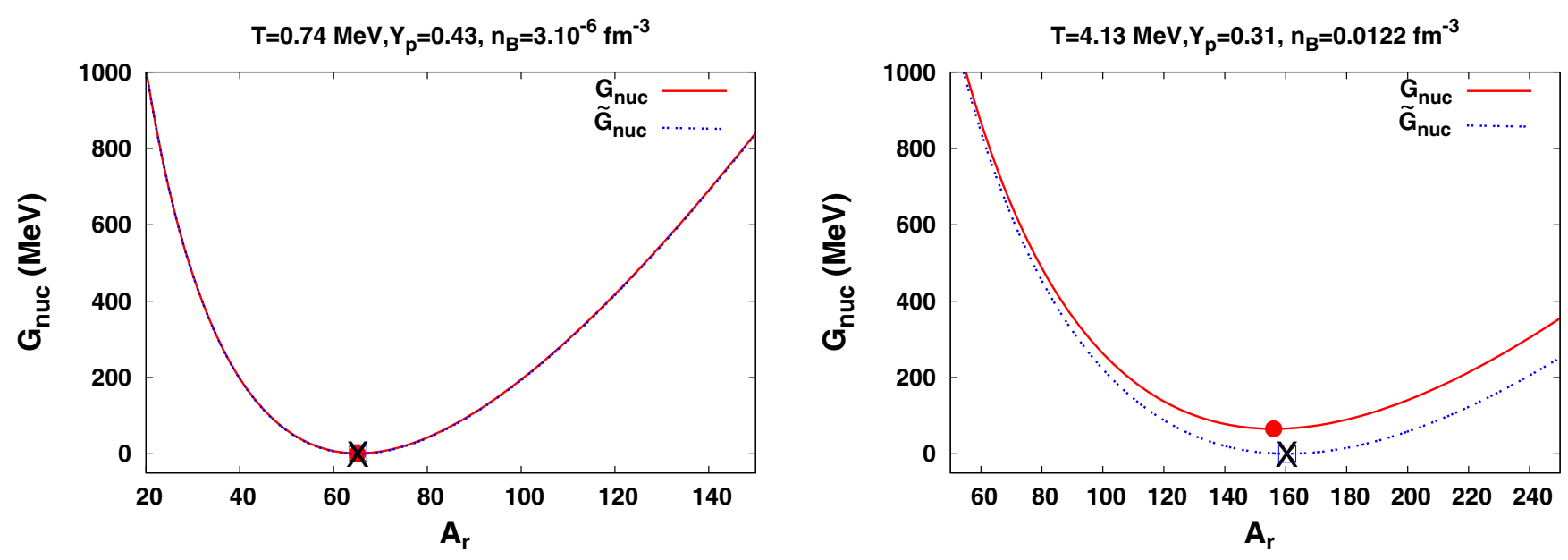

FIG. 1. Nuclear contribution to the Gibbs free energy, with and without the rearrangement term, for two different thermodynamic conditions, as a function of the number of nucleons in the dense phase $A_{r}: n_{B}=3 \times 10^{-6} \mathrm{fm}^{-3}, T=0.74 \mathrm{MeV}, Y_{e}=Y_{p}=0.43$ (left panel) and $n_{B}=$ $1.22 \times 10^{-2} \mathrm{fm}^{-3}, T=4.13 \mathrm{MeV}, Y_{e}=Y_{p}=0.31$ (right panel). The fraction $x_{i}=Z_{r} / A_{r}$ is fixed to the one of the most probable nucleus. The dots (squares) correspond to the most probable nucleus obtained without (with) the rearrangement term; the crosses indicate the $A_{r}$ of the average nucleus obtained with the LS EoS. See text for details.

$h\left(x_{i}, T\right)$ is a temperature-dependent function entering in the surface free energy (see Eq. (2.28) in [8]), and $\mu_{H}$ is the translational contribution to the chemical potential (see Eq. (2.43) in [8]).

(ii) $\left.\frac{\partial \tilde{G}_{\text {nuc }}}{\partial x_{i}}\right|_{n_{i}, r_{N}}=0$, that gives

$$
\begin{aligned}
& \mu_{n i}-\mu_{p i}-\frac{2}{3} \frac{\mathscr{D} \beta}{n_{i} u}\left(\frac{1}{x_{i}}+\frac{\sigma^{\prime}}{\sigma}\right)-\frac{1-u}{A_{0}} h^{\prime}\left(\mu_{H}-T\right) \\
& =\mu_{n o}-\mu_{p o}
\end{aligned}
$$

where $\sigma \equiv \sigma\left(x_{i}, T\right)$ entering the surface term of the free energy is defined in Eq. (2.27) in LS [8], $\sigma^{\prime} \equiv \partial \sigma / \partial x_{i}$, and $h^{\prime} \equiv \partial h / \partial x_{i}$. Note that combining Eqs. (40) and (41) yields Eqs. (3.2b), (3.2c) in [8], see Appendix B.

(iii) $\left.\frac{\partial \tilde{G}_{\text {nuc }}}{\partial r_{N}}\right|_{n_{i}, x_{i}}=0$. Noting that minimizing $\tilde{G}_{\text {nuc }}^{(j)}$ with respect to $r_{N}$ is equivalent to minimize $\tilde{G}_{\text {nuc }}^{(j)}$ with respect to the nuclear volume $V_{N}$ yields

$$
\frac{\partial\left(\mathcal{F}_{s}+\mathcal{F}_{c}\right)}{\partial V_{N}}=0 \quad \& \quad \frac{G_{\mathrm{nuc}}}{V_{N}}+\left\langle\frac{u}{V_{N}} \frac{\partial G_{\mathrm{nuc}}^{(j)}}{\partial u}\right\rangle=0 .
$$

The first condition in Eq. (42) gives the equilibrium equation for $r_{N}$, or, equivalently, for $n_{i}$ [see Eq. (2.38) in LS [8] and Appendix B, Eq. (B9)],

$$
n_{i}=\frac{5}{2} \frac{A_{r} \sigma}{Z_{r}^{2} e^{2}}\left(\frac{u(1-u)}{\mathscr{D}}\right)^{3},
$$

$e$ being the elementary charge. Using Eq. (40) and the expression for $\mu_{\alpha}$, Eq. (B11), together with the thermodynamic relation among the pressure $P$, the free energy density $\mathcal{F}$, and the chemical potential $\mu$ of a species with density $n, P=-\mathcal{F}+\mu n$, applied to the pressure of the gas, $P_{o}$, that of the alpha particles, $P_{\alpha}$, and that of the nucleons inside the nucleus, $P_{i}$, the second condition in Eq. (42) becomes

$$
P_{i}+\beta\left(\frac{2 \mathscr{D}}{3 u}-\mathscr{D}^{\prime}\right)+\frac{n_{i} u}{A_{0}} h \mu_{H}=P_{o}+P_{\alpha}
$$

with $\mathscr{D}^{\prime} \equiv \partial \mathscr{D} / \partial u$. This expression is equivalent to Eq. (3.2d) of LS [8], see Appendix B, Eq. (B14). Note that the term accounting for the binding energy of $\alpha$ particles, $B_{\alpha}$, does not appear in our Eq. (B11) since the chemical potentials in our definition include the rest mass. The introduction of the rearrangement term is indeed required to get the equivalence of the results obtained in the canonical and grand-canonical approach, and therefore the thermodynamic consistency of the approach.

The effect of the rearrangement term is analyzed in Fig. 1, where we display the nuclear part of the Gibbs free energy, Eq. (38), for two typical conditions of density, temperature, and proton fraction encountered in the collapsing core of a supernova. The minimum of each curve represents the most probable cluster, obtained with or without the rearrangement term. The crosses correspond to the average single nucleus calculated in the LS EoS, while the dot (square) symbols mark the most probable nucleus calculated with the NSE approach without (with) the rearrangement term. The latter acts in a more significant way at higher density and temperature. Indeed, while no noticeable difference is observed at lower densities (left panel), only including this term allows one to correctly reproduce the LS most probable nucleus for higher densities (right panel).

\section{RESULTS}

We present in this section the results obtained with the extended NSE model, implemented in perturbation on the top 


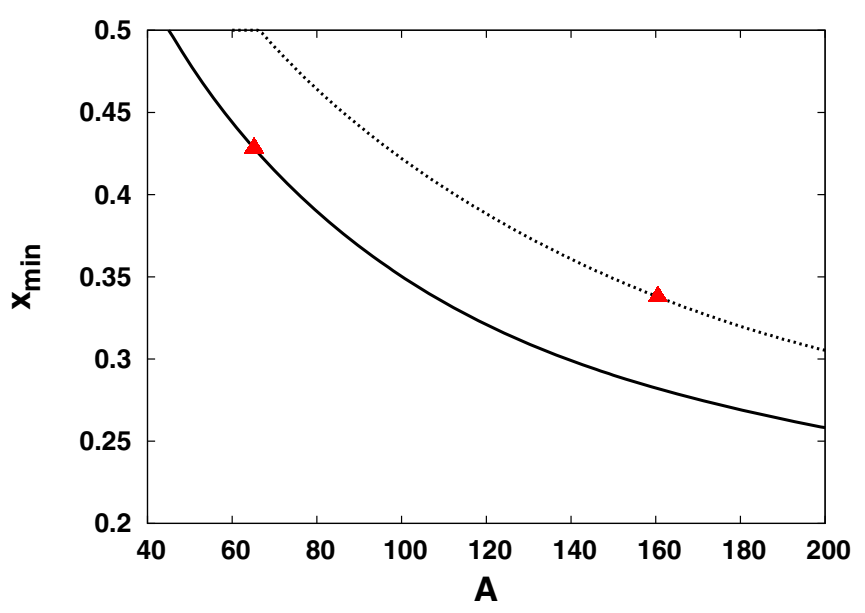

FIG. 2. Ratio $x_{i}=Z / A$ corresponding to the minimum of $\tilde{G}_{\text {nuc }}$ at a fixed $A$, as a function of $A$, for two thermodynamic conditions: $n_{B}=3 \times 10^{-6} \mathrm{fm}^{-3}, T=0.74 \mathrm{MeV}, Y_{e}=0.43$ (solid line) and $n_{B}=1.22 \times 10^{-2} \mathrm{fm}^{-3}, T=4.13 \mathrm{MeV}, Y_{e}=0.31$ (dashed line). Triangles indicate the values obtained with the LS EoS.

of the LS EoS. This means that, for a given thermodynamic condition, the calculation of the EoS and composition is done in two steps: (i) first, we run the LS analytical routine thus obtaining the SNA results, (ii) then, varying $A$ and $Z$, we calculate $n_{i}$ solving Eq. (43) and $\tilde{G}_{\text {nuc }}(A, Z)$, Eq. (38), as explained in Sec. II, thus the probability $p(A, Z)$, Eq. (25), associated to each cluster $(A, Z) .^{2}$ In this way, we can also compare the most probable nucleus in the NSE model (i.e., the one that minimizes $\tilde{G}_{\text {nuc }}$ ) with the SNA one. The resolution of Eq. (43) for $n_{i}$ is done using the root finder routine broydn from the Numerical Recipes [58], which employs the Broyden's method. ${ }^{3}$

We start by showing, in Fig. 2, the $x_{i}=Z / A$ ratio corresponding to the minimum of $\tilde{G}_{\text {nuc }}$, as a function of $A$. As an illustrative example, we have chosen two thermodynamic conditions likely to be met during the infall phase of the supernova core collapse: $n_{B}=3 \times 10^{-6} \mathrm{fm}^{-3}, T=0.74 \mathrm{MeV}$, $Y_{e}=0.43$ (solid line) and $n_{B}=1.22 \times 10^{-2} \mathrm{fm}^{-3}, T=$ $4.13 \mathrm{MeV}, Y_{e}=0.31$ (dashed line). These numbers, as well as other conditions explored below, are taken from a collapse trajectory followed by the innermost region of the star. The thermodynamic conditions for the latter have been obtained with a one-dimensional hydrodynamic code in general relativity with a neutrino leakage-type scheme [59-61], starting from a $40 M_{\odot}$ progenitor from Woosley et al. [62]. The considered collapse trajectory stops before bounce, before nuclei dissociate in favor of homogeneous matter. Triangles indicate the $\left(x_{i}, A\right)$ values

\footnotetext{
${ }^{2}$ In this section, in the spirit of the LS EoS, we define $A$ and $Z$ as the baryon and proton numbers of the dense phase, $A=V_{N} n_{i}, Z=A x_{i}$.

${ }^{3}$ Note that the solution may be sensitive to the convergence criterion, thus the numerical precision required in the root finder. Although we have found that the resulting value of the most probable $Z$ or $N$ may vary up to two units for a few of the thermodynamic conditions explored below, the robustness of our results is not affected and our conclusions remain unchanged.
}

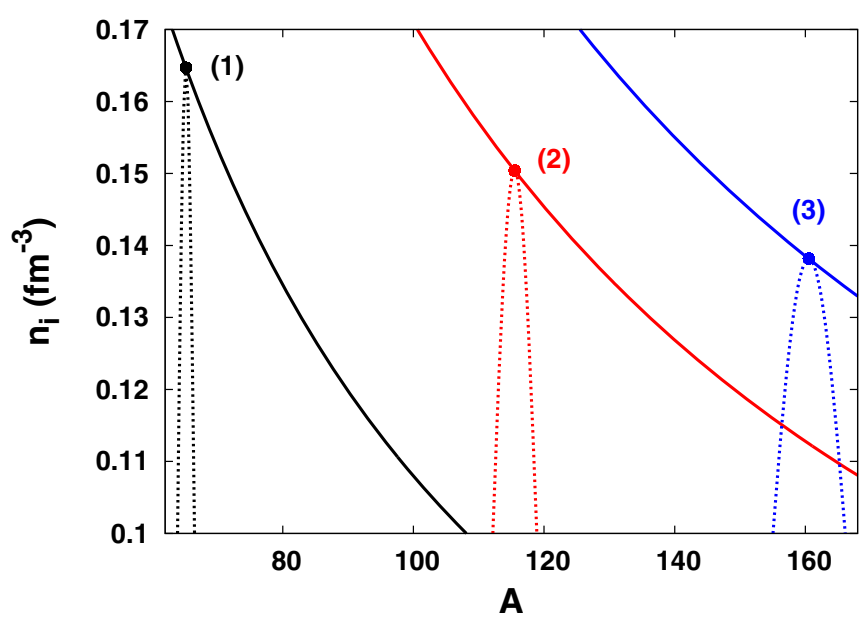

FIG. 3. Baryon number density inside the nucleus, $n_{i}$, versus $A$ for three thermodynamic conditions: (1) $n_{B}=3 \times 10^{-6} \mathrm{fm}^{-3}, T=$ $0.74 \mathrm{MeV}, Y_{e}=0.43$, (2) $n_{B}=2 \times 10^{-3} \mathrm{fm}^{-3}, T=2.3 \mathrm{MeV}, Y_{e}=$ 0.34 , and (3) $n_{B}=1.22 \times 10^{-2} \mathrm{fm}^{-3}, T=4.13 \mathrm{MeV}, Y_{e}=0.31$. Solid lines correspond to the solution of Eq. (43), dots correspond to $n_{i}$ for the average nucleus predicted by the LS EoS, and dashed lines represent the cluster distribution (in arbitrary units).

obtained from the original LS model (that is, within the SNA) for the same thermodynamic conditions: $x_{i, \mathrm{LS}}=0.43, A_{\mathrm{LS}}=$ 65 and $x_{i, \mathrm{LS}}=0.34, A_{\mathrm{LS}}=161$, respectively. One can notice that the behavior of $x_{i}$ is sensitive to the nuclear distribution, changing of about $50 \%$ when $A$ varies from about 50 to 200 , and it can be quite different from the value predicted by the LS for the same input variables $\left(n_{B}, T, Y_{e}\right)$, when the nuclear mass number $A$ is different from the LS one.

In Fig. 3, we plot the number density inside the nucleus, $n_{i}$, as calculated by solving Eq. (43), versus $A$, for a fixed $x_{i}=\left(x_{i}\right)_{\text {SNA }}$ equal to that obtained by the LS EoS, for three representative thermodynamic conditions taken from the collapse trajectory: (1) $n_{B}=3 \times 10^{-6} \mathrm{fm}^{-3}, T=0.74 \mathrm{MeV}, Y_{e}=$ 0.43 , (2) $n_{B}=2 \times 10^{-3} \mathrm{fm}^{-3}, T=2.3 \mathrm{MeV}, Y_{e}=0.34$, and (3) $n_{B}=1.22 \times 10^{-2} \mathrm{fm}^{-3}, T=4.13 \mathrm{MeV}, Y_{e}=0.31$. Dashed lines represent the cluster distribution (in arbitrary units), while dots correspond to $\left(n_{i}\right)_{\mathrm{SNA}}$ for the average single nucleus predicted by the LS EoS. We notice that the most probable nucleus, which is identified by the peak of the cluster distribution, coincides with that predicted by the LS EoS, both having the same density $n_{i}$, for the three conditions. We also note that the density inside the nucleus decreases with $A$; because of mass conservation, Eq. (B5), this is compensated by an increase of the density of the outside gas, $n_{o}$, and that of $\alpha$ particles, $n_{\alpha}$. Moreover, increasing density and temperature, from condition (1) to (3), yields to an increase of the average cluster mass number and of the width of the distribution.

To better compare the NSE results with the SNA ones, we show in Fig. 4 the cluster $Z$ versus $N$ distribution (panel b), for a chosen thermodynamic condition: $n_{B}=8.6 \times 10^{-4} \mathrm{fm}^{-3}, T=1.83 \mathrm{MeV}, Y_{e}=0.36$. The contour plot displays the cluster probabilities (red to blue, more to less abundant), while the black dot corresponds to the LS average nucleus, $Z_{\mathrm{SNA}}=38, N_{\mathrm{SNA}}=62$. This shows 


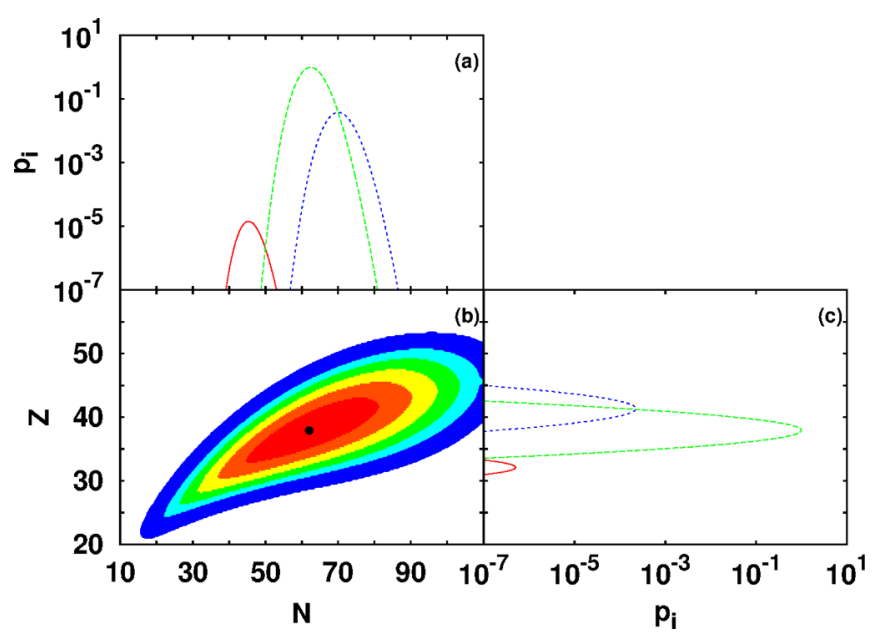

FIG. 4. Distribution of nuclei predicted by the NSE model for the thermodynamic condition $n_{B}=8.6 \times 10^{-4} \mathrm{fm}^{-3}, T=1.83 \mathrm{MeV}$, $Y_{e}=0.36$. (b) shows the contour plot of the cluster probabilities (red to blue, from more to less abundant), while the black dot corresponds to the average nucleus $\left(Z_{\mathrm{SNA}}=38, N_{\mathrm{SNA}}=62\right)$ obtained with the LS EoS. (a) displays the probability distributions for $Z=32,38$, and 41 (from left to right curve), while (c) displays the probability distribution for $N=40,62$, and 80 (from bottom to top curve).

that our extended NSE reproduces the SNA results, having the advantage to take into account a full distribution of nuclei. The smooth cluster distribution with a single-centered peak also points out the absence of shell effects. Panels (a) and (c) illustrate the probability distribution, $p(A, Z)$ (in arbitrary units), for different cuts in $Z=32,38,41$ [panel (a), from left to right], and $N=40,62,80$ [panel (c), from bottom to top], respectively. The center (green) curves in panels (a) and (c) correspond to the most probable nucleus, which coincides again with the SNA result.

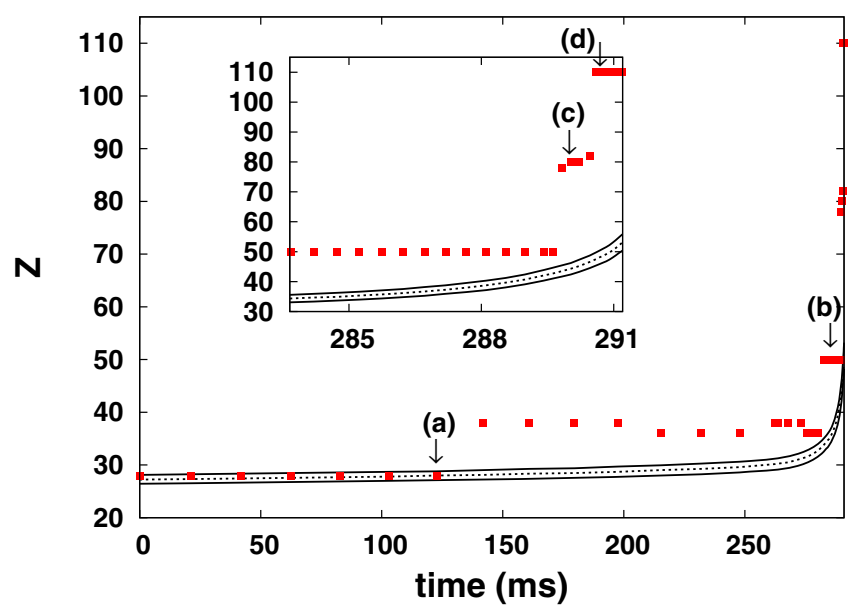

\section{A. Inclusion of a microscopic nuclear mass model}

One of the limit of the compressible liquid-drop model employed in the LS EoS is the absence of pairing and shell effects, that are responsible for the emergence of magic numbers and impact the cluster distribution, especially at relatively low density and temperature (see, e.g., Refs. [38,47,52]). Indeed, shell and pairing effects are expected to be washed out only at a temperature of a few $\mathrm{MeV}$. To take these effects into account, either experimental or theoretical mass models have to be implemented. However, in core-collapse supernovae, the electron capture drives nuclei towards the neutron-rich side, where experimental mass measurements are no longer available and theoretical extrapolations need to be considered. Therefore, we have included in our perturbative extended NSE nuclear binding energies from a theoretical mass model. To this aim, we have redefined the cluster Gibbs free energy, replacing the contribution corresponding to the nuclear binding energy in the compressible liquid-drop model, $G_{\mathrm{LS}}^{0}$, with the theoretical calculated binding energy, $E_{\text {bind }}$ :

$$
\tilde{G}_{\mathrm{nuc}}=\tilde{G}_{\mathrm{nuc}, \mathrm{LS}}-G_{\mathrm{nuc}, \mathrm{LS}}^{0}+E_{\mathrm{bind}},
$$

where $\tilde{G}_{\text {nuc,LS }}$ corresponds to the nuclear contribution to the Gibbs free energy as calculated in Eq. (38), and $G_{\text {nuc,LS }}^{0}$ is the same quantity evaluated at $T=0$ and with $n_{o}=n_{\alpha}=$ $n_{e}=0$ ( $n_{e}$ being the electron number density), i.e., the bulk plus surface (at zero temperature) and Coulomb (without the screening correction) term. As for the theoretical mass model, we have employed the Brussels-Montreal microscopic mass model $^{4}$ HFB-24 [63]. This model, based on the self-consistent Hartree-Fock-Bogoliubov method using a 16-parameter generalized Skyrme effective nucleon-nucleon interaction with a realistic contact pairing force, fits the 2353 measured masses of

${ }^{4}$ The mass table for this model is available on the BRUSLIB database at http://www.astro.ulb.ac.be/bruslib/.

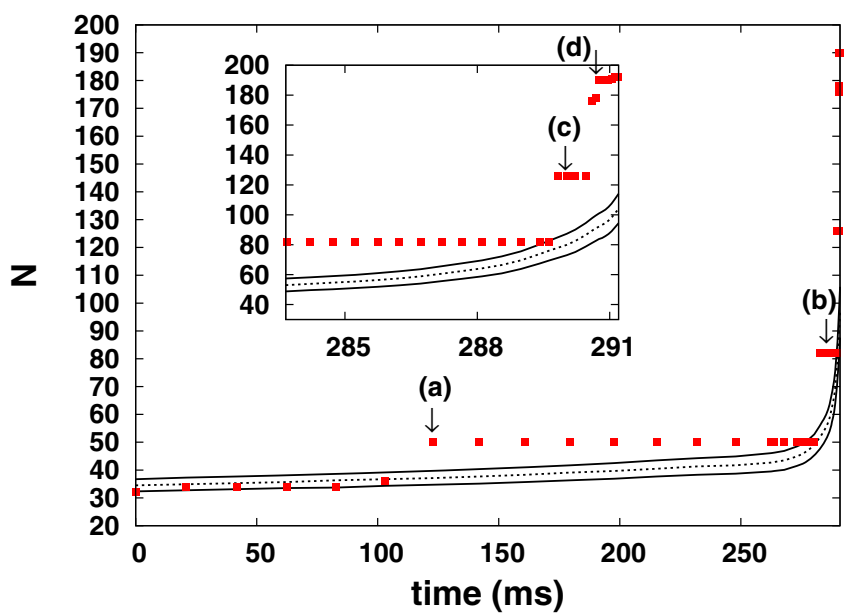

FIG. 5. $Z$ (left panel) and $N$ (right panel) along the collapse trajectory (time is given in milliseconds), at the center of the star. Dashed lines correspond to the most probable $Z$ (left panel) and $N$ (right panel) obtained with the LS mass functional, while solid lines correspond to the variance. Red dots represent the most probable $Z$ (left panel) and $N$ (right panel) obtained implementing the HFB-24 mass model. Insets show a zoom of the final part of the plot. Arrows indicate four chosen points along the trajectory before the core bounce. See text for details. 
nuclei with $N$ and $Z \geqslant 8$ appearing in the 2012 Atomic Mass Evaluation (AME) [64] with a root-mean square deviation of $0.5 \mathrm{MeV}$. Moreover, it is also compatible with other various experimental and astrophysical constraints [65]. Note that, in the mass table, atomic mass excess are tabulated; once atomic masses, $M_{\text {at }}(A, Z)$, are known, nuclear masses, $M_{\text {nuc }}$, can be calculated as

$$
M_{\mathrm{nuc}}(A, Z)=M_{\mathrm{at}}-Z m_{e} c^{2}+B_{e l}(Z),
$$

where $m_{e}$ is the electron mass and $B_{e l}(Z)$ is the total binding energy of all $Z$ removed electrons (see Appendix $\mathrm{A}$ in Ref. [66]).

To illustrate the effect of employing such a mass table instead of the simplified LS functional on the composition during the supernova core collapse, we have plotted in Fig. 5 the evolution of $Z$ (left panel) and $N$ (right panel) for given thermodynamic conditions along the collapse trajectory in the center of the star; the increasing time (given in milliseconds) on the $x$ axis corresponds to a simultaneous increase of density
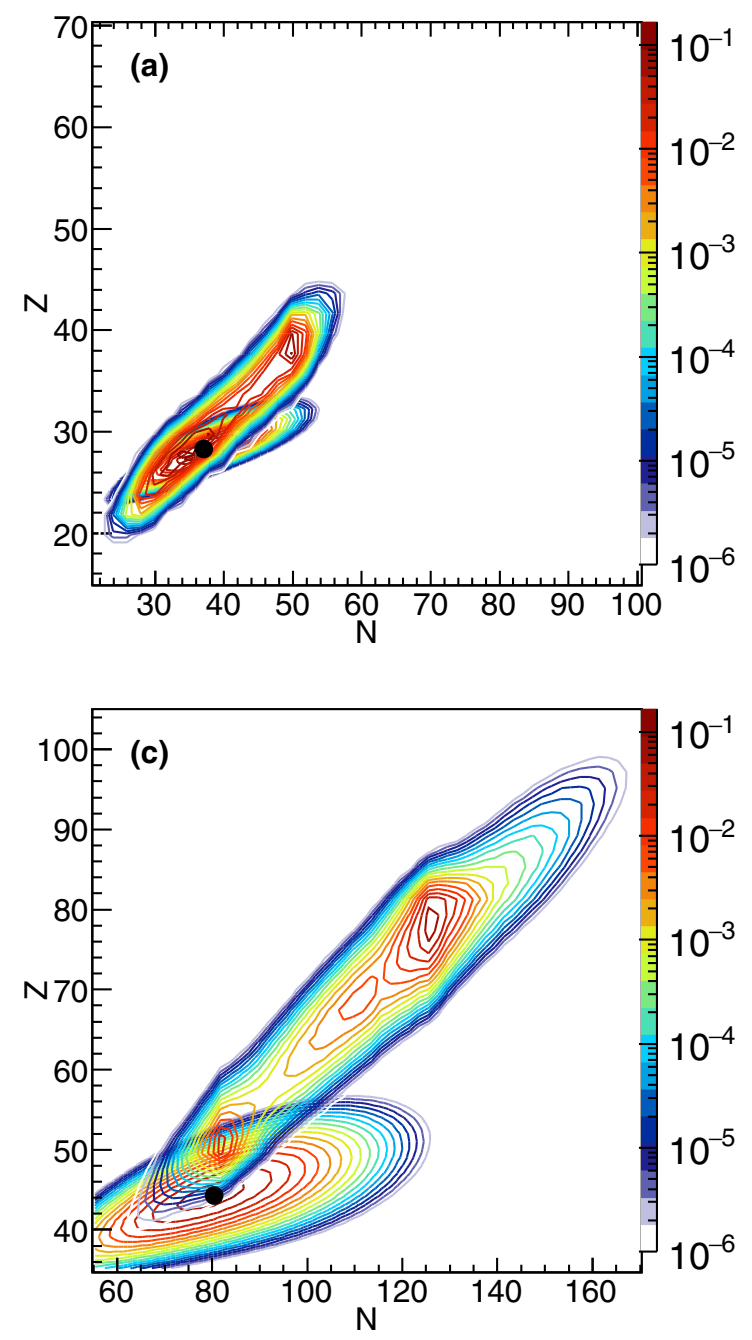

and temperature. The dashed lines correspond to the most probable $Z$ (left panel) and $N$ (right panel) calculated with the extended NSE model built on the top of the original LS EoS, thus keeping the nuclear masses as in the original compressible liquid-drop model; solid lines represent the variance of $Z$ and $N$ and give the width of the distribution. We note that the spread in the nuclear distribution increases with time, i.e., with density and temperature. The most probable $Z(N)$ obtained when the HFB-24 mass model is employed instead of the original mass model implemented in the LS EoS are displayed by dots in the left (right) panel. We can clearly notice the emergence of shell structure and magic numbers: in the first phase of collapse, clusters with $Z=28$ dominate, while in subsequent stages of collapse, clusters with $N$ around 50 first and then with $N=82,126$ prevail. At this point of collapse, remarkable differences in the composition can be observed when one or the other model is used. Indeed, as already noticed, e.g., in Ref. [52], the use of different mass models and the presence (or absence) of shell effects impact
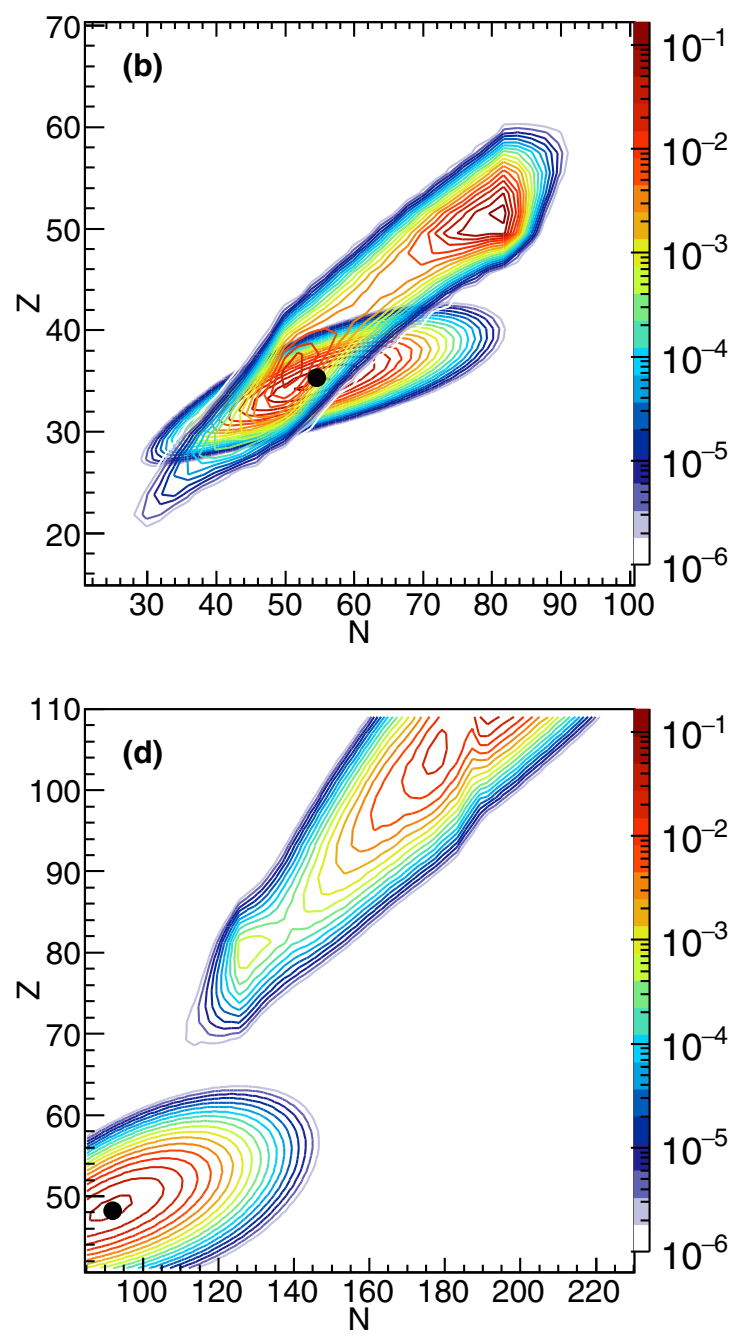

FIG. 6. Distribution of nuclei $(N, Z)$ for four chosen thermodynamic conditions along a collapse trajectory at the center of the star: (a) $n_{B}=3.86 \times 10^{-6} \mathrm{fm}^{-3}, T=0.79 \mathrm{MeV}, Y_{e}=0.43$, (b) $n_{B}=3.35 \times 10^{-4} \mathrm{fm}^{-3}, T=1.51 \mathrm{MeV}, Y_{e}=0.38,(\mathrm{c}) n_{B}=3.01 \times 10^{-3} \mathrm{fm}{ }^{-3}$, $T=2.68 \mathrm{MeV}, Y_{e}=0.33$, and (d) $n_{B}=5.85 \times 10^{-3} \mathrm{fm}^{-3}, T=3.33 \mathrm{MeV}, Y_{e}=0.31$. Contour lines correspond to the cluster normalized probabilities (red to blue, more to less probable) for the original LS model and for the HFB-24 nuclear mass model. Dots correspond to the average single nucleus predicted by the LS EoS. See text for details. 
in a considerable way the prediction of the composition in NSE models. The four arrows in Fig. 5 labeled from (a) to (d) point at four chosen instants along the trajectory, before bounce, whose thermodynamic conditions $\left(n_{B}, T, Y_{e}\right)$ correspond to the nuclear distributions shown in the four panels of Fig. 6: (a) $n_{B}=3.86 \times 10^{-6} \mathrm{fm}^{-3}, T=0.79 \mathrm{MeV}, Y_{e}=$ 0.43 , (b) $n_{B}=3.35 \times 10^{-4} \mathrm{fm}^{-3}, T=1.51 \mathrm{MeV}, Y_{e}=0.38$, (c) $n_{B}=3.01 \times 10^{-3} \mathrm{fm}^{-3}, T=2.68 \mathrm{MeV}, Y_{e}=0.33$, and (d) $n_{B}=5.85 \times 10^{-3} \mathrm{fm}^{-3}, T=3.33 \mathrm{MeV}, Y_{e}=0.31$. The colored contour lines in Fig. 6 (red to blue, more to less probable nuclei) represent the cluster normalized probability, Eq. (25), obtained (i) with the original LS model, where the nuclear masses are taken from the original compressible liquid-drop model employed in the LS EoS (note that the oval-shaped distributions are indeed centered on the SNA results, represented by the black dots) and (ii) with the HFB-24 mass model. It is also clear from this figure the appearance of magic numbers when the HFB-24 mass model is employed. Indeed, in this case, a bimodal distribution, which cannot be reproduced in the single-peaked SNA picture, emerges. While for thermodynamic conditions at the beginning of the collapse (panel a) nuclei around $Z=28$ and $N=50$ are populated, later on (panel b), the distribution is peaked around $N=50$ and $N=82$, and subsequently (panel c) around $N=82$ and $N=126$; towards the end of the infall phase (panel d), the most probable nuclei are probably situated around $N=184$. We can also notice that, along the collapse, the most probable nuclei become bigger (i.e., $A$ increases), and the distributions become larger. These effects are a consequence of the increasing density and temperature, respectively.

Finally, an important difference is observed in panels (c) and (d) between the average nucleus predicted by the original LS EoS (dots) and the most probable nuclei predicted using the HFB-24 nuclear mass models. This indicates that the latter application with the HFB model, contrary to the previous one where the mass model was taken from the LS EoS, is not fully consistent. To achieve consistency, we should calculate the chemical potentials $\mu_{q o}$ and the full $\tilde{G}_{\text {nuc }}$ functional directly from the same energy-density functional as the one used to compute the nuclear masses. This improvement is left for future work.

\section{CONCLUSIONS}

In this paper, we have worked out a general formalism allowing to calculate the nuclear distribution encountered in equilibrium stellar matter, associated to any arbitrary unified EoS, that is a finite temperature nuclear modeling able to describe both homogeneous and inhomogeneous asymmetric nuclear matter. The cluster probabilities are given by simple Boltzmann factors, Eq. (25), with four important modifications:

(1) the cluster size entering in the Legendre transformation from Helmotz to Gibbs free energy must be properly defined such as to ensure mass and charge conservation, see Eqs. (6),(7);

(2) the cluster chemical potential is given by the chemical potential of the unbound nucleons, see Eqs. (A8),(A9), multiplied by the effective cluster size;
(3) all eventual in-medium modifications must be considered inside the cluster free energy, see Eq. (4) and the following discussion;

(4) a rearrangement term insuring thermodynamical consistency must be added, see Eq. (24).

This general formalism can be straightforwardly applied to any relativistic or nonrelativistic subsaturation EoS based on the WS approximation, that is within the SNA. We have applied it to the specific case of the LS EoS, which is widely used in core-collapse simulations. This example has explicitly shown that the most probable nucleus coincides with the prediction of the LS EoS, but many other nuclei are populated with a nonnegligible probability, and the width of the nuclear distribution is an increasing function of the density and temperature.

We have also explored the effect of using a more realistic mass model, by replacing the simpler compressible liquid-drop like functional for nuclear masses in the LS EoS with the more microscopic Brussels-Montreal HFB-24 mass model, which provides an excellent reproduction of nuclear masses all over the nuclear chart.

Considering thermodynamic conditions followed by a typical core-collapse trajectory, we have shown that the isotopic distribution considerably deviates from the average LS results, and magic nuclei dominate the whole trajectory. Since the electron-capture rates on nuclei may vary of orders of magnitude among neighboring nuclei, we expect that a consistent calculation of the rates over this more realistic distribution may change the deleptonization rates, and thus the resulting dynamics of the collapse, with respect to standard collapse calculations where the SNA is employed. We emphasize that, especially for high densities and temperatures where the contribution of the gas surrounding the clusters increases, the neutron and proton chemical potentials of the gas should be calculated from the same theoretical model used to compute the nuclear masses. This is indeed necessary to achieve full consistency in the model. These calculations, as well as the implementation of the electron-capture rates on the nuclear distribution and the evaluation of the effect on the collapse trajectory, is left for future work.

\section{ACKNOWLEDGMENTS}

G.G. was supported by CAPES/COFECUB agreement no. $\mathrm{Ph}$ 853-15. Partial support from the COST Action MP1304 "NewCompStar" is gratefully acknowledged.

\section{APPENDIX A: CHEMICAL POTENTIALS}

In this appendix, we show that it is possible to identify the Lagrange multipliers, introduced to impose the constraints on the mass and charge conservation, and the chemical potentials introduced in the expression of the Gibbs free energy, with the chemical potentials of the homogeneous matter (i.e., of the uniform gas). Let us consider the case where matter is modeled with only one type of WS cells, for simplicity. Starting from the baryon free energy in the WS cell,

$$
F_{\mathrm{bar}}=F_{\mathrm{nuc}}-k_{B} T V_{C} \ln z_{\beta},
$$


where $k_{B}$ is the Boltzmann constant and the free energy density of the gas is $\mathcal{F}_{g}=-k_{B} T \ln z_{\beta}, z_{\beta}$ being the meanfield partition sum for homogeneous matter, one can write the Gibbs free energy of the baryon component operating a Legendre transformation with respect to the neutron and proton baryon numbers (or, equivalently, the total baryon number and isospin). One thus gets

$$
G_{\mathrm{bar}}=F_{\mathrm{bar}}-\mu_{1} N_{\mathrm{WS}}-\mu_{2} Z_{\mathrm{Ws}},
$$

where we have introduced the auxiliary chemical potentials $\mu_{1}$ and $\mu_{2}$. Using Eqs. (6),(7) [where we have dropped the $(j)$ in the notation for convenience], we obtain

$$
G_{\text {bar }}=F_{\text {bar }}-\mu_{1}\left(N+n_{n g} V_{C}\right)-\mu_{2}\left(Z+n_{p g} V_{C}\right) .
$$

Using the mean-field relations of uniform nuclear matter,

$$
\ln z_{\beta \mu_{n} \mu_{p}}=\ln z_{\beta}+\beta \mu_{1} n_{n g}+\beta \mu_{2} n_{p g},
$$

with $\beta=\left(k_{B} T\right)^{-1}$, one can see that the neutron and proton gas densities are given by

$$
\begin{aligned}
& n_{n g}=\left.k_{B} T \frac{\partial \ln z_{\beta \mu_{n} \mu_{p}}}{\partial \mu_{1}}\right|_{\mu_{2}}, \\
& n_{p g}=\left.k_{B} T \frac{\partial \ln z_{\beta \mu_{n} \mu_{p}}}{\partial \mu_{2}}\right|_{\mu_{1}} .
\end{aligned}
$$

Therefore, the gas densities are uniquely determined by the chemical potentials, and independent on the realization $(k)$, i.e., $n_{n g}^{(k)}=n_{n g}$ and $n_{p g}^{(k)}=n_{p g}$. The Gibbs free energy is finally given by

$$
\begin{aligned}
G_{\mathrm{bar}} & =F_{\text {nuc }}-\mu_{1} N-\mu_{2} Z-k_{B} T V_{C} \ln z_{\beta \mu_{n} \mu_{p}} \\
& =G_{\text {nuc }}+V_{C} \mathcal{G}_{g}
\end{aligned}
$$

with $\mathcal{G}_{g}$ the Gibbs free energy density of the gas, and

$$
\begin{aligned}
& \mu_{1}=-\left.k_{B} T \frac{\partial \ln z_{\beta}}{\partial n_{n g}}\right|_{n_{p g}}=\mu_{n g}, \\
& \mu_{2}=-\left.k_{B} T \frac{\partial \ln z_{\beta}}{\partial n_{p g}}\right|_{n_{n g}}=\mu_{p g},
\end{aligned}
$$

where one recognizes that the auxiliary chemical potentials coincide with the neutron and proton chemical potentials of the homogeneous gas, $\mu_{n} \equiv \mu_{n g}$ and $\mu_{p} \equiv \mu_{p g}$.

\section{APPENDIX B: LS EOS IN THE CANONICAL ENSEMBLE}

In this appendix we rederive the equilibrium equations, Eqs. (3.2) in LS [8], that result from the minimization of the total free energy density of the system. The procedure is equivalent to minimize the auxiliary function constructed from the free energy density under conservation constraints (see also Ref. [47]), yielding the SNA results. Since the lepton (and photon) contribution to the total free energy density can be treated separately, and does not enter in the minimization, we only consider here the (total) baryon free energy density. In the LS EoS, the chosen set of independent variables are $\left\{x_{k}\right\}=$ $\left\{n_{i}, x_{i}, u, r_{N}, n_{\alpha}, n_{n o}, n_{p o}\right\}$, where $n_{i}=A_{r} / V_{N}$ and $x_{i}=Z_{r} / A_{r}$ are the nucleon density and proton fraction inside the nucleus in the cell, respectively, $u=V_{N} / V_{C}$ is the fraction of the WS cell volume occupied by nuclei $\left(V_{N}\right.$ being the volume of the nucleus), $r_{N}$ is the nuclear radius (such that $V_{N}=4 \pi r_{N}^{3} / 3$ ), $n_{\alpha}, n_{n o}$, and $n_{p o}$ are the number density of the $\alpha$ particles, and of the neutrons and protons in the outside gas, respectively. For simplicity of notation, we omit in this section the superscript $(j)$ referring to the cell. Using these LS variables, the baryon free energy density can be written as

$$
\mathcal{F}_{\text {bar }}=\mathcal{F}_{\text {nuc }}+\mathcal{F}_{o}+\mathcal{F}_{\alpha} \text {. }
$$

The nuclear contribution is given by the sum of the bulk, surface, Coulomb, and translational free energy density,

$$
\begin{aligned}
\mathcal{F}_{\text {nuc }}= & n_{i} u\left[f_{b}\left(n_{i}, x_{i}\right)+f_{s}\left(x_{i}, u, r_{N}\right)+f_{c}\left(x_{i}, n_{i}, u, r_{N}\right)\right] \\
& +n_{i} u(1-u) f_{H}\left(n_{i}, x_{i}, u\right),
\end{aligned}
$$

where we have put in evidence the dependences on the independent variables (see Ref. [8] for the detailed expressions of the different terms). Note that, in the present notation, $f$ corresponds to free energy per baryon, while $\mathcal{F}$ to free energy density. For the nucleons in the outside gas and the alpha particles, we have

$$
\begin{aligned}
& \mathcal{F}_{o}=n_{o} f_{o}\left(n_{o}\right)(1-u)\left(1-n_{\alpha} v_{\alpha}\right), \\
& \mathcal{F}_{\alpha}=n_{\alpha} f_{\alpha}\left(n_{\alpha}\right)(1-u)
\end{aligned}
$$

with $n_{o}=n_{n o}+n_{p o}$.

The mass and charge conservations read

$$
\begin{aligned}
n_{B}= & \frac{A_{\mathrm{WS}}}{V_{C}}=u n_{i}+4 n_{\alpha}(1-u) \\
& +\left(n_{n o}+n_{p o}\right)(1-u)\left(1-n_{\alpha} v_{\alpha}\right), \\
n_{p}= & n_{e}=\frac{Z_{\mathrm{WS}}}{V_{C}}=x_{i} n_{i} u+2 n_{\alpha}(1-u) \\
& +n_{p o}(1-u)\left(1-n_{\alpha} v_{\alpha}\right),
\end{aligned}
$$

where $A_{\mathrm{WS}}$ and $Z_{\mathrm{WS}}$ are the total baryon and proton number in the cell, respectively (the total neutron number in the cell is thus $N_{\mathrm{WS}}=A_{\mathrm{WS}}-Z_{\mathrm{WS}}$, and a conservation for $n_{n}$ can be derived, too) and $v_{\alpha}=24 \mathrm{fm}^{3}$ is the volume occupied by each $\alpha$ particle. One can then construct the auxiliary function to be minimized:

$$
\begin{aligned}
\mathcal{D}= & \mathcal{F}_{\text {bar }}-\lambda_{1}\left[n_{p}-x_{i} n_{i} u-2 n_{\alpha}(1-u)\right. \\
& \left.-n_{p o}(1-u)\left(1-n_{\alpha} v_{\alpha}\right)\right] \\
& -\lambda_{2}\left[n_{n}-\left(1-x_{i}\right) n_{i} u-2 n_{\alpha}(1-u)\right. \\
& \left.-n_{n o}(1-u)\left(1-n_{\alpha} v_{\alpha}\right)\right],
\end{aligned}
$$

where $\lambda_{1}$ and $\lambda_{2}$ are the Lagrange multipliers. The minimization of the auxiliary function, Eq. (B7), with respect to the seven variables yields

(i) $\left.\frac{\partial \mathcal{D}}{\partial r_{N}}\right|_{n_{i}, x_{i}, u, n_{\alpha}, n_{n o}, n_{p o}}=0$. Noting that only the surface and Coulomb terms depend on $r_{N}$, i.e., $\partial\left(\mathcal{F}_{s}+\right.$ $\left.\mathcal{F}_{c}\right) / \partial r_{N}=0$, one recovers the virial theorem, $\mathcal{F}_{s}=$ $2 \mathcal{F}_{c}$, that gives the equation to be solved separately for the nuclear radius, Eq. (3.2a) in [8],

$$
r_{N}=\frac{9 \sigma}{2 \beta}\left(\frac{s(u)}{c(u)}\right)^{1 / 3}
$$


where $s(u)$ and $c(u)$ are shape parameters aiming to mimic the transition from nuclei to the bubble phase [8]. Equivalently, one can write Eq. (B8) in terms of $n_{i}$

$$
n_{i}=\frac{5}{2} \frac{A_{r} \sigma}{Z_{r}^{2} e^{2}} \frac{s(u)}{c(u)} .
$$

Note that in the LS routine, $s(u) / c(u)=[u(1-$ u) $/ \mathscr{D}]^{3}$.

(ii) $\left.\frac{\partial \mathcal{D}}{\partial n_{q o}}\right|_{n_{i}, x_{i}, u, r_{N}, n_{\alpha}, n_{-q o}}=0$, with $q=n, p$ and $-q=$ $p, n$. This condition identifies the Lagrange multipliers as the neutron and proton chemical potentials,

$$
\lambda_{1}=-\mu_{p o} \quad \text { and } \quad \lambda_{2}=-\mu_{n o} .
$$

(iii) $\left.\frac{\partial \mathcal{D}}{\partial n_{\alpha}}\right|_{n_{i}, x_{i}, u, r_{N}, n_{n o}, n_{p o}}=0$. Using the thermodynamic relation between the pressure $P$, the free energy density $\mathcal{F}$, and the chemical potential $\mu$ of a species of density $n, P=-\mathcal{F}+\mu n$, this condition gives Eq. (3.2e) of $\mathrm{LS}^{5}$

$$
\mu_{\alpha}=2\left(\mu_{n o}+\mu_{p o}\right)-v_{\alpha} P_{o},
$$

where $P_{o}$ is the pressure of the neutron and proton gas.

\footnotetext{
${ }^{5}$ Note that in Eq. (3.2e) in [8] the minus sign between the neutron and proton chemical potentials should be replaced with a plus sign. Moreover, $B_{\alpha}$ is not included in Eq. (B11) because masses are included in the chemical potentials.
}

(iv) $\left.\frac{\partial \mathcal{D}}{\partial x_{i}}\right|_{n_{i}, u, r_{N}, n_{\alpha}, n_{n o}, n_{p o}}=0$, that gives Eq. (3.2b) of LS,

$$
\begin{aligned}
& \mu_{n i}-\mu_{p i}-\frac{2}{3} \frac{\beta \mathscr{D}}{n_{i} u}\left(\frac{1}{x_{i}}+\frac{\sigma^{\prime}}{\sigma}\right)-\frac{1-u}{A_{0}} h^{\prime}\left(\mu_{H}-T\right) \\
& =\mu_{n o}-\mu_{p o},
\end{aligned}
$$

with $\sigma^{\prime} \equiv \partial \sigma / \partial x_{i}$ and $h^{\prime} \equiv \partial h / \partial x_{i}$.

(v) $\left.\frac{\partial \mathcal{D}}{\partial n_{i}}\right|_{x_{i}, u, r_{N}, n_{\alpha}, n_{n o}, n_{p o}}=0$.

(vi) $\left.\frac{\partial \mathcal{D}}{\partial u}\right|_{x_{i}, n_{i}, r_{N}, n_{\alpha}, n_{n o}, n_{p o}}=0$

Combining the last two conditions with Eq. (B12), and noting that $\mu_{b, i}=\left(1-x_{i}\right) \mu_{n i}+x_{i} \mu_{p i}$ after operating the change of variables $\left(n_{n i}, n_{p i}\right) \rightarrow\left(n_{i}, x_{i}\right)$, with $n_{n i}=n_{i}\left(1-x_{i}\right)$ and $n_{p i}=n_{i} x_{i}$, gives Eqs. (3.2c),(3.2d) of LS

$$
\begin{aligned}
\mu_{n i} & -\frac{2}{3} \frac{x_{i} \beta \mathscr{D}}{n_{i} u} \frac{\sigma^{\prime}}{\sigma}+\frac{1-u}{A_{0}}\left[h \mu_{H}-h^{\prime} x_{i}\left(\mu_{H}-T\right)\right] \\
& =\mu_{n o}, \\
P_{i} & +\beta\left(\frac{2}{3} \frac{\mathscr{D}}{u}-\mathscr{D}^{\prime}\right)+\frac{n_{i} h}{A_{0}} \mu_{H} u \\
= & P_{o}+P_{\alpha},
\end{aligned}
$$

where $\mathscr{D}^{\prime} \equiv \partial \mathscr{D} / \partial u$.
[1] G. Baym, C. Pethick, and P. Sutherland, Astrophys. J. 170, 299 (1971).

[2] G. Baym, H. A. Bethe, and C. J. Pethick, Nucl. Phys. A 175, 225 (1971).

[3] D. G. Ravenhall, C. J. Pethick, and J. R. Wilson, Phys. Rev. Lett. 50, 2066 (1983).

[4] M. Hashimoto, H. Seki, and M. Yamada, Prog. Theor. Phys. 71, 320 (1984).

[5] H. A. Bethe, G. E. Brown, J. Applegate, and J. M. Lattimer, Nucl. Phys. A 324, 487 (1979).

[6] J. Cooperstein, Nucl. Phys. A 438, 722 (1985).

[7] J. M. Lattimer, C. J. Pethick, D. G. Ravenhall, and D. Q. Lamb, Nucl. Phys. A 432, 646 (1985).

[8] J. M. Lattimer and F. Douglas Swesty, Nucl. Phys. A 535, 331 (1991).

[9] A. da Silva Schneider, L. F. Roberts, and C. D. Ott, Phys. Rev. C 96, 065802 (2017).

[10] M. Lassaut, H. Flocard, P. Bonche, P.-H. Heenen, and E. Suraud, Astron. Astrophys. 183, L3 (1987).

[11] M. Onsi, H. Przysiezniak, and J. M. Pearson, Phys. Rev. C 55, 3139 (1997).

[12] H. Shen, H. Toki, K. Oyamatsu, and K. Sumiyoshi, Nucl. Phys. A 637, 435 (1998).

[13] H. Shen, H. Toki, K. Oyamatsu, and K. Sumiyoshi, Prog. Theor. Phys. 100, 1013 (1998).
[14] H. Shen, H. Toki, K. Oyamatsu, and K. Sumiyoshi, Astrophys. J. Suppl. Ser. 197, 20 (2011).

[15] M. Onsi, A. K. Dutta, H. Chatri, S. Goriely, N. Chamel, and J. M. Pearson, Phys. Rev. C 77, 065805 (2008).

[16] S. S. Avancini, D. P. Menezes, M. D. Alloy, J. R. Marinelli, M. M. W. Moraes, and C. Providência, Phys. Rev. C 78, 015802 (2008).

[17] S. S. Avancini, S. Chiacchiera, D. P. Menezes, and C. Providência, Phys. Rev. C 82, 055807 (2010).

[18] S. S. Avancini, C. C. Barros, Jr., L. Brito, S. Chiacchiera, D. P. Menezes, and C. Providência, Phys. Rev. C 85, 035806 (2012).

[19] F. Grill, H. Pais, C. Providência, I. Vidaña, and S. S. Avancini, Phys. Rev. C 90, 045803 (2014).

[20] Z. W. Zhang and H. Shen, Astrophys. J. 788, 185 (2014).

[21] H. Pais, S. Chiacchiera, and C. Providência, Phys. Rev. C 91, 055801 (2015).

[22] P. Bonche and D. Vautherin, Nucl. Phys. A 372, 496 (1981).

[23] P. Bonche and D. Vautherin, Astron. Astrophys. 112, 268 (1982).

[24] P. Gögelein, E. N. E. van Dalen, C. Fuchs, and H. Müther, Phys. Rev. C 77, 025802 (2008).

[25] N. Sandulescu, Phys. Rev. C 70, 025801 (2004).

[26] H. Pais and J. R. Stone, Phys. Rev. Lett. 109, 151101 (2012).

[27] A. Pastore, Phys. Rev. C 86, 065802 (2012).

[28] M. Oertel, M. Hempel, T. Klähn, and S. Typel, Rev. Mod. Phys. 89, 015007 (2017). 
[29] A. Burrows and J. M. Lattimer, Astrophys. J. 285, 294 (1984).

[30] A. Juodagalvis, K. Langanke, W. R. Hix, G. Martinez-Pinedo, and J. M. Sampaio, Nucl. Phys. A 848, 454 (2010).

[31] C. Sullivan, E. O'Connor, R. G. T. Zegers, T. Grubb, and S. M. Austin, Astrophys. J. 816, 44 (2016).

[32] Ad. R. Raduta, F. Gulminelli, and M. Oertel, Phys. Rev. C 95, 025805 (2017).

[33] S. Furusawa, H. Nagakura, K. Sumiyoshi, C. Kato, and S. Yamada, Phys. Rev. C 95, 025809 (2017).

[34] M. F. El Eid and W. Hillebrandt, Astron. Astrophys. Suppl. Ser. 42, 215 (1980).

[35] W. Hillebrandt, K. Nomoto, and R. G. Wolff, Astron. Astrophys. 133, 175 (1984).

[36] S. Heckel, P. P. Schneider, and A. Sedrakian, Phys. Rev. C 80, 015805 (2009).

[37] A. S. Botvina and I. N. Mishustin, Nucl. Phys. A 843, 98 (2010).

[38] M. Hempel and J. Schaffner-Bielich, Nucl. Phys. A 837, 210 (2010).

[39] Ad. R. Raduta and F. Gulminelli, Phys. Rev. C 82, 065801 (2010).

[40] S. I. Blinnikov, I. V. Panov, M. A. Rudzsky, and K. Sumiyoshi, Astron. Astrophys. 535, A37 (2011).

[41] S. Furusawa, S. Yamada, K. Sumiyoshi, and H. Suzuki, Astrophys. J. 738, 178 (2011).

[42] M. Hempel, T. Fischer, J. Schaffner-Bielich, and M. Liebendörfer, Astrophys. J. 748, 70 (2012).

[43] S. Furusawa, K. Sumiyoshi, S. Yamada, and H. Suzuki, Astrophys. J. 772, 95 (2013).

[44] A. W. Steiner, M. Hempel, and T. Fischer, Astrophys. J. 774, 17 (2013).

[45] N. Buyukcizmeci, A. S. Botvina, and I. N. Mishustin, Astrophys. J. 789, 33 (2014).

[46] Ad. R. Raduta, F. Aymard, and F. Gulminelli, Eur. Phys. J. A 50, 24 (2014).

[47] F. Gulminelli and A. R. Raduta, Phys. Rev. C 92, 055803 (2015).

[48] S. Burrello, F. Gulminelli, F. Aymard, M. Colonna, and Ad. R. Raduta, Phys. Rev. C 92, 055804 (2015).

[49] S. Furusawa, K. Sumiyoshi, S. Yamada, and H. Suzuki, Nucl. Phys. A 957, 188 (2017).

[50] C. Iliadis, Nuclear Physics of Stars (Wiley-VCH, New York, 2007).
[51] A. C. Phillips, The Physics of Stars (Wiley \& Sons, Chichester, UK, 1999).

[52] N. Buyukcizmeci, A. S. Botvina, I. N. Mishustin et al., Nucl. Phys. A 907, 13 (2013).

[53] A. Weiss, W. Hillebrandt, H.-C. Thomas, and H. Ritter, Cox and Giuli's Principles of Stellar Structure, extended 2nd ed. (Cambridge Scientific Publishers, Cambridge, 2004).

[54] P. Haensel, A. Y. Potekhin, and D. G. Yakovlev, Neutron Stars 1: Equation of State and Structure (Springer, Berlin, 2007).

[55] J. P. Bondorf, A. S. Botvina, A. S. Iljinov, I. N. Mishustin, and K. Sneppen, Phys. Rep. 257, 133 (1995).

[56] R. Buras, M. Rampp, H.-T. Janka, and K. Kifonidis, Astron. Astrophys. 447, 1049 (2006).

[57] M. Oertel, A. F. Fantina, and J. Novak, Phys. Rev. C 85, 055806 (2012).

[58] W. H. Press, B. P. Flannery, S. A. Teukolsky, and W. T. Vetterling, Numerical Recipes in FORTRAN 77: The Art of Scientific Computing, 2nd ed. (Cambridge University Press, Cambridge, 1997).

[59] J. V. Romero, J. M. A. Ibáñez, J. M. A. Marti, and J. A. Miralles, Astrophys. J. 462, 839 (1996).

[60] J. V. Romero, J. M. Ibáñez, J. A. Miralles, and J. A. Pons, in Some Topics on general relativity and gravitational radiation, edited by J. A. Miralles, J. A. Morales, and D. Sáez (Editions Frontieres, Paris, 1997), p. 289.

[61] A. F. Fantina, Supernovae theory: Study of electro-weak processes during gravitational collapse of massive stars, Ph.D. thesis, Université Paris XI, Orsay, France, and Università degli Studi di Milano, Milano, Italy (2010).

[62] S. E. Woosley, A. Heger, and T. A. Weaver, Rev. Mod. Phys. 74, 1015 (2002).

[63] S. Goriely, N. Chamel, and J. M. Pearson, Phys. Rev. C 88, 024308 (2013).

[64] G. Audi, M. Wang, A. H. Wapstra, F. G. Kondev, M. MacCormick, X. Xu, and B. Pfeiffer, Chin. Phys. C 36, 1287 (2012).

[65] J. M. Pearson, N. Chamel, A. F. Fantina, and S. Goriely, Eur. Phys. J. A 50, 43 (2014).

[66] D. Lunney, J. M. Pearson, and C. Thibault, Rev. Mod. Phys. 75, 1021 (2003). 